\section{Fundamentos y perspectivas \\ desde donde pensar la integración \\ de la docencia y la extensión en la Universidad Nacional del Litoral}

\section{Mariana Boffelli}

Docente investigadora y coordinadora del Área de Incorporación Curricular de la Extensión de la Secretaría de Extensión de la Universidad Nacional del Litoral, Argentina.

boffelli@fbcb.unl.edu.ar

\section{Sandra Sordo}

Docente del Instituto Superio del Profesorado $n^{\circ} 64$ Santo Tomé, Argentina.

Integrante del equipo del Área de Incorporación Curricular de la Extensión de la Secretaría de Extensión de la Universidad Nacional del Litoral. ssordo@rectorado.unl.edu.ar

\section{Resumen}

El artículo que se presenta aquí pretende dar cuenta del trabajo realizado por el Área de Incorporación Curricular de la Extensión de la Secretaría de Extensión de la Universidad Nacional del Litoral y las perspectivas teóricas que sustentan un enfoque particular, como lo es la educación experiencial. En este sentido, se intenta compartir, respecto de sus distintas secciones, desde qué perspectivas y cómo se viene trabajando la integración de la extensión en el currículo universitario, partiendo de las dimensiones que dan lugar a una definición de extensión y, con relación a esto, las tensiones y desafíos que implica pararnos en un marco más amplio.

Se trata de visibilizar la experiencia llevada a cabo por el área en el Plan y Acción: Fortalecimiento de la Educación Experiencial en el currículo universitario, enmarcado en el Plan de Desarrollo Institucional de la universidad (2010-2019).
Integración de la docencia y la extensión /

Perspectivas

RECEPCIÓN: 24/06/16
ACEPTACIÓN FINAL: 10/08/16

\section{Resumo}

O artigo aqui apresentado tenta explicar o trabalho feito pela Departamento de Área de Integração Curricular da Extensão da Secretaria de Extensão da Universidade Nacional del Litoral e perspectivas teóricas que sustentam um enfoque particular, como é a educação experiencial. Nesse sentido, tenta-se compartilhar, a respeito de suas diferentes seções, de que perspectivas e como se vem trabalhando a integração da extensão no currículo universitário, partindo das dimensões que dão lugar a uma definição de extensão e, no que diz a respeito disto, as tensões e desafios que exige posicionar-nos em um cenário mais amplo.

Pretende-se tornar visível a experiência levada a cabo pela área no Plano e Ação: Fortalecimento da Educação Experiencial no currículo universitário, pertencente ao Plano de Desenvolvimento Institucional da universidade (2010-2019).

Palavras-chave

- Integração docência- extensão

- Educação experiencial

- Universidade Nacional del Litoral
- Integración docencia-extensión

- Educación experiencial

- Universidad Nacional del Litoral

Para citación de este artículo Boffelli, M. y Sordo, S. (2016). Fundamentos y perspectivas desde donde pensar la integración de la docencia y la extensión. En Revista $+E$ versión digital, (6), pp.16-23. Santa Fe, Argentina: Ediciones UNL 


\section{6}

Se puede observar que

hay dificultades en identificar

las fuentes de la producción

del conocimiento

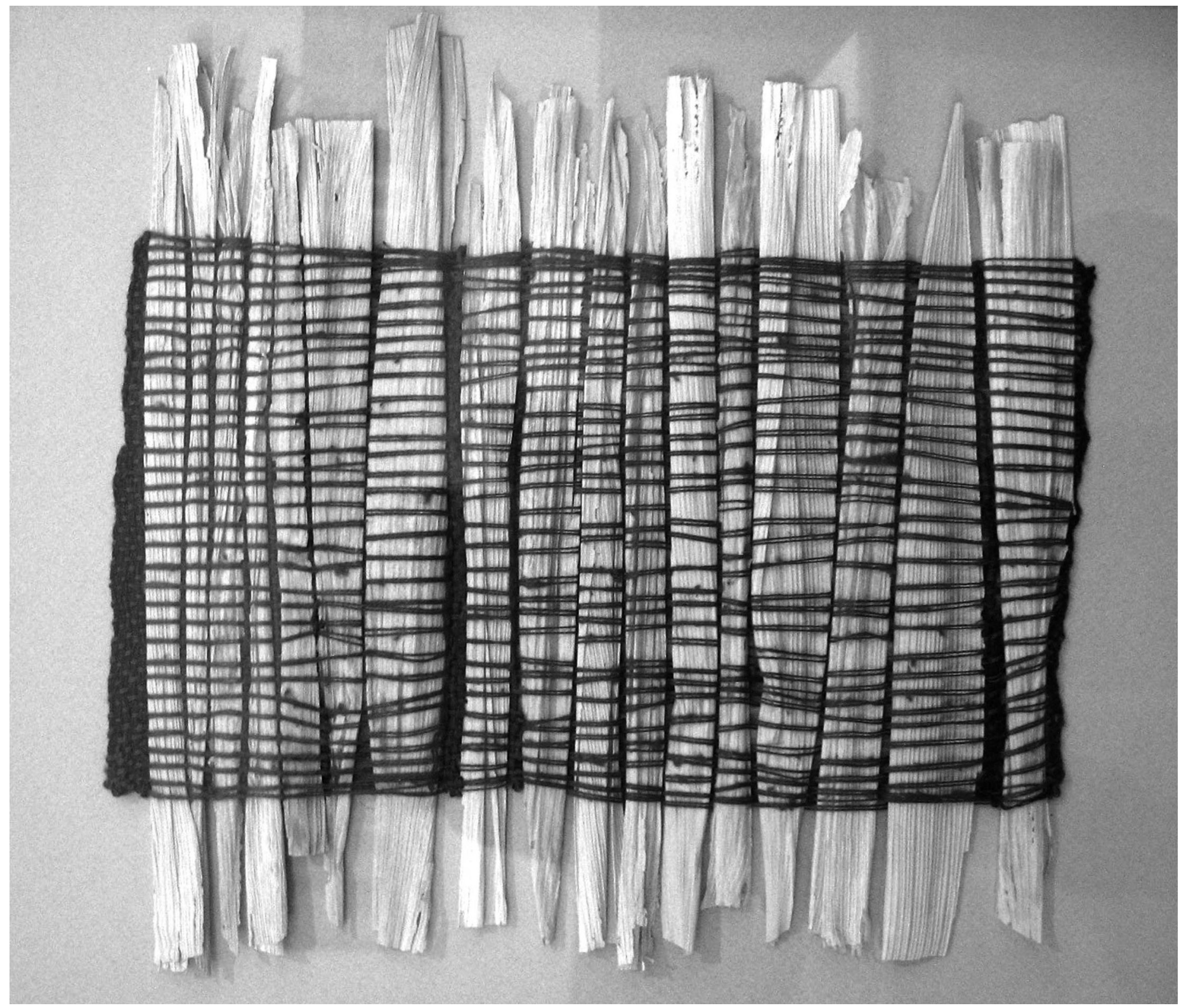

(c) Cecilia lucci 


\section{Introducción}

El desafío tiene que ver con pensar el diseño de mecanismos de implementación, a partir de la Resolución CS n² 274/07, que plasma el reto asumido institucionalmente de fomentar la incorporación de prácticas de extensión en las carreras de grado de la Universidad Nacional del Litoral (UNL).

La UNL es una institución pública de Educación Superior que proyecta su labor de enseñanza, investigación y extensión y que no se limita a la formación de grado sino que se retroalimenta con las exigencias del contexto para enriquecer estas funciones. Considerando el tema que nos ocupa, la extensión y la docencia, haremos una breve mención, en primer término, de los alcances del concepto de extensión que aparecen de los diferentes documentos institucionales elaborados por esta Casa de Estudios para luego colocarlo en relación con la docencia. Entre estos documentos encontramos: "Educación y ciencia como proyecto político" (2005), que destaca el nuevo significado que adquiere la extensión para el siglo XXI; "Un aporte desde las políticas de extensión universitaria" (2006), que concibe a la extensión como una "interfaz" entre Universidad y la sociedad que promueve la construcción de un "nuevo entorno de cooperación e influencia mutua"; "Plan de Desarrollo Institucional” (PDI), aprobado por Asamblea Universitaria correspondiente al período 2010-2019 donde se destaca que:

"la extensión es una función esencial de nuestra Universidad que promueve el diálogo permanente con el Estado —en sus diferentes jurisdicciones- con otras instituciones educativas y científico tecnológicas del sistema provincial y nacional, con las organizaciones de la sociedad civil y de la producción en la búsqueda de soluciones a los problemas del contexto, lo que confiere a estas acciones una alta pertinencia social (...) [y donde se resalta que] la integración entre la Extensión y la Enseñanza nos posiciona desde una perspectiva en la que los saberes culturales y los conocimientos académicos-científicos se ponen en juego con otros saberes y conocimientos que posee la sociedad, lo que posibilita analizar y abordar los problemas a partir de una concepción y comprensión de la complejidad." (UNL, 2010:20)

A partir de los documentos apuntados, se brindan elementos que configuran el posible marco en el cual pensar la extensión en nuestra Universidad y, en ese sentido, creemos que es posible enfocarla desde múltiples dimensiones: académico-institucional, social, comunicacional, política y pedagógica de la extensión.

\section{Las dimensiones de la extensión}

Hablar de la dimensión sustantiva e institucional es hablar de la relevancia académica que ha adquirido la extensión universitaria a lo largo del tiempo, lo que le permitió constituirse en uno de los pilares fundamentales donde se construye un modelo de universidad que tiene su referencia histórica en el modelo de la Reforma Universitaria de 1918. Un modelo que, más allá de las diferencias y particularidades, considera a la educación superior como un derecho humano fundamental y una función indelegable del Estado, guiada por los principios de democracia, autonomía y pensamiento crítico, de compromiso social y calidad académica, tal como lo expresa la declaración de Cartagena de Indias de Educación Superior para Latinoamérica y el Caribe pronunciada en el año 2008. Reconocer una dimensión social en la extensión universitaria significa poder pensar esta función sustantiva como promotora de un desarrollo humano y sustentable y que a su vez sea un vehículo para la construcción de espacios sociales. Desde la perspectiva de Max Neef, pensar en estrategias de desarrollo significa analizar el binomio necesidades-satisfactores. Las necesidades consideradas en una doble dimensión: como carencias y como potencialidad. Los satisfactores constituyen la dimensión histórica de las necesidades, desde una doble trayectoria: se modifican con el ritmo de las transformaciones, se diversifican acordes a la cultura y los estratos sociales. Son formas de ser, tener, hacer y estar. Incluyen formas de organización, estructuras políticas, prácticas sociales y condiciones subjetivas.

Max Neef concibe un modelo de desarrollo que promueve la satisfacción de necesidades humanas fundamentales, que genere crecientes niveles de autodependencia, una articulación armónica de los seres humanos con la naturaleza y la tecnología, que articule procesos globales con las particularidades locales, lo personal con lo social, y que incluya mecanismos de participación que fortalezcan la vinculación Estado y sociedad civil.

Por otra parte, la extensión es pensada también como una herramienta para la construcción de espacios sociales, y es en ellos donde se ponen en acción y en cuestión las producciones teóricas con la realidad, que posibilitan de este modo la construcción de conocimientos que sean útiles para todos los actores sociales involucrados. Alfredo Carballeda (2002) analiza el escenario de la intervención en lo social y expresa que ésta se configura en un contexto de crisis que, durante los últimos 30 años, ha dificultado el sostenimiento de lazos sociales y derivó en un proceso de "fragmentación social". De esta manera, la intervención debe ser un mecanismo de integración.

En tanto, se reconoce en la extensión una "dimensión comunicacional", planteada en términos de diálogo. Dicho en palabras de Paulo Freire:

"la comunicación verdadera no es la transferencia, o la transmisión del conocimiento, de un sujeto a otro, sino su coparticipación en el acto de comprender la significación del significado. Es una comunicación que se hace críticamente" (1973:78-79). 
Lo que caracteriza a la comunicación es que ella es diálogo, así como el diálogo es comunicativo, y para que eso exista es necesario que los sujetos, recíprocamente comunicantes, estén de acuerdo; por lo que el contenido del conocimiento extendido se debe originar en un terreno común a los polos de la relación, en un proceso, como sujetos del conocimiento y no como receptores del mismo. Las prácticas sociales, como dice Teresa Matus, deben estar iluminadas por el tramado discursivo, que nos sitúa en el plano del mundo de la vida; por lo tanto, se hace necesario volcar una hermenéutica viable que resuelva esta relación de interpretación y comprensión de la realidad (Matus, 2005).

También reconocer la dimensión política: como lo expresa el documento institucional "La intervención social en el marco de la Extensión Universitaria":

"La intervención social, en el marco de las acciones de extensión, refiere tanto a una dimensión territorial, como institucional, íntimamente vinculada a las políticas públicas. De allí que la identificación y análisis de las mismas, permite reconocer las prioridades que el Estado establece; reconocer los actores sociales que participan en las mismas; el perfil de sus beneficiarios; analizar en qué medida reflejan y responden a las demandas y necesidades ciudadanas; su incidencia en procesos de inclusión social, como indicador relevante de la sustentabilidad." (Kessler y otros, 2016:2)

Y por último, se reconoce en la extensión una "dimensión pedagógica" que creemos contribuye significativamente en los procesos de enseñanza y de aprendizaje. Parafraseando a Alicia Camilloni:

"El éxito en el logro de estos propósitos requiere que los currículos y las estrategias de enseñanza sean exitosos en la promoción de aprendizajes auténticos, esto es, aquellos aprendizajes en los que los estudiantes construyen conocimientos "estratégicos" o "condicionales" que implican saber por qué, dónde, cuándo y cómo se utilizan esos conocimientos, elaborados, particularmente, en relación con situaciones, casos, problemas o proyectos tal y como se encuentran en la vida real." (2013:11)

Diseñar prácticas de extensión integradas al currículo universitario significa problematizar y resignificar, desde otro lugar, las prácticas de enseñanza y posibilitar otra forma de aprender. Este tipo de experiencia deberá permitir a los estudiantes universitarios, futuros profesionales, la oportunidad de involucrarse en la resolución de problemas reales de la comunidad, lo que implica trabajar en escenarios reales con problemas complejos, por lo que deben ser abordados desde una perspectiva interdisciplinar.

Diseñar una práctica de este tipo supone integrar contextos, contenidos curriculares, maneras de enseñar y de aprender, la formación profesional y cómo evaluar el aprendizaje en ese proceso. Entonces, hablar de "prácticas" vinculadas a la extensión como función sustantiva de la institución y como componente de la estructura curricular de las carreras estaría vinculado con la dimensión pedagógica, la noción de "interacción" relacionado con la dimensión comunicacional, y la "intervención" conectada con la dimensión social y política.

Dicho esto, consideramos relevante recuperar las ideas de algunos autores cuyos aportes se vinculan con la temática que aquí pretendemos poner en discusión, la incorporación curricular de la extensión en las carreras de grado (de la UNL), esto es, la posibilidad de pensar en la extensión como una valiosa herramienta que permita promover el aprendizaje en el marco de las carreras universitarias.

\section{Integrar la extensión al currículo universitario}

Según Sousa Santos (2008), la universidad en la actualidad se enfrenta a una situación compleja en los países latinoamericanos: la sociedad le hace exigencias cada vez mayores, al mismo tiempo que se hacen cada vez más restringidas las políticas de financiamiento de sus actividades por parte del Estado. Doblemente desafiada por la sociedad y el Estado, la universidad debe enfrentar esos retos. El autor confiere centralidad a las actividades de extensión (con implicancia en el currículo y en la carrera docente) y atribuye a la universidad una participación activa en la construcción de la cohesión social, profundización de la democracia, la lucha contra la exclusión social y la defensa de la diversidad cultural.

Cabe considerar las nociones que plantea el autor con respecto a la producción y reproducción de conocimiento, proceso que implica a varios actores, como la universidad, la sociedad y el Estado, donde las acciones se materializan en estructuras que aportan a complejizar lo que Alicia De Alba señala en la noción de curriculum como práctica social:

"Por curriculum se entiende a la síntesis de elementos culturales (conocimientos, valores, costumbres, creencias, hábitos) que conforman una propuesta político-educativa pensada e impulsada por diversos grupos y sectores sociales cuyos intereses son diversos y contradictorios, aunque algunos tienden a ser dominantes o hegemónicos, y otros tienden a oponerse y resistirse a tal dominación o hegemonía." (1995:59)

Se puede observar que hay dificultades en identificar las fuentes de la producción del conocimiento. Por eso se señala que los alumnos en determinadas oportunidades consideran que el conocimiento se logra sólo mediante la asimilación de teorías, y en otros casos que el aprendizaje sólo toma fuerza, se actualiza y toma vigencia, en la práctica cotidiana. Es por eso que hay que tener en cuenta la importancia de las actitudes. Toda persona observadora puede advertir cada día, tanto en sí misma como en los demás, cierta tendencia a creer en aquello que visualiza más con sus deseos. La capacidad para educar el pensamiento no se 
consigue simplemente a través del conocimiento de las mejores formas de pensamiento, se tiene que dar la unión de la actitud con el método. Es decir, lo que se debe hacer es cultivar las actitudes, como es, entre otras: una mentalidad abierta. Tal como lo advierte John Dewey, esta mentalidad:

"puede definirse como carencia de prejuicios, y cualquier hábito que limite la mente y le impida considerar nuevos problemas y asumir nuevas ideas (...) Incluye un deseo de escuchar a más de una parte, de acoger los hechos con independencia de su fuente, de restar atención, a las posibilidades alternativas, de reconocer la posibilidad de error incluso respecto de las creencias que apreciamos más (...) cultivar la curiosidad constante y el espontáneo esfuerzo de comprensión de todo lo nuevo, que constituye la esencia de ésta actitud"; otra es el entusiasmo, "cuando alguien se interesa vigorosamente (...) las preguntas se les ocurren espontáneamente, se insinúan y se realizan nuevas investigaciones y lecturas, el material atrae y estimula la mente del sujeto y comunica un renovado ímpetu a su pensamiento. El auténtico entusiasmo (...) es una actitud que actúa como una fuerza intelectual"; la responsabilidad actitud necesaria para adquirir un apoyo adecuado para la búsqueda de nuevos puntos de vista e ideas (...). Ser intelectualmente responsable quiere decir considerar las consecuencias de un paso proyectado. (...) Las tres actitudes que se han mencionado, son en sí mismas cualidades personales, rasgos del carácter y son elementos constitutivos esenciales de esta disposición general". (1960:43-44)

Ahora bien, no podemos dejar de compartir con Félix Temporetti las siguientes preguntas:

“¿Quién aprende?, ¿cómo se concibe a los alumnos/as o aprendices?, ¿cómo individuos (sujetos) concretos, inteligentes, encarnados, sexuados, históricos y situados en una cultura que transforman y en buena medida los constituye?, ¿cómo poseedores de un sistema o mecanismo inteligente, general y abstracto, que los habilita para aprender o procesar la información?" (2006:98)

La educación experiencial es una teoría pedagógica desarrollada por David Kolb (1984) en su libro La educación experiencial implica un ciclo. Este enfoque, en términos del autor, entiende al aprendizaje como el proceso por el cual el conocimiento es creado a través de la transformación de la experiencia. Esta teoría tiene una base fuerte en la obra de John Dewey (1938), filósofo de la educación del siglo XX:

"el principio de la continuidad de la Experiencia significa que cada experiencia tanto ocupa algo de los que se han ido antes y modifica de alguna manera la calidad de los que vienen después (...). Lo que ha aprendido en el camino del conocimiento y habilidad en una situación se convierte en un instrumento de entender y tratar eficazmente con las situaciones que siguen. El proceso continúa mientras la vida y el aprendizaje continúan." (Citado por Kolb, 1984:27)

Las Prácticas de Extensión de Educación Experiencial (PEEE) se constituyen para la Universidad Nacional del Litoral en un dispositivo de intervención en lo social que:

"se presenta como un instrumento de transformación no solo de las circunstancias donde concretamente actúa, sino también como un dispositivo de integración y facilitación del diálogo entre diferentes lógicas que surgen de distinta forma comprensiva explicativa, no solo de los problemas sociales, sino de las Instituciones en sí mismas." (Carballeda, 2002:3-4)

La intervención social que proponen las PEEE ancla en una construcción de conocimiento, en términos de Carballeda, no a priori sino a posteriori. Eso implica un conocimiento que se produce desde el hacer mismo, es decir, el estudiante aprende en situación. Esta intervención se funda en una concepción alejada de la enseñanza de meros contenidos teóricos y de aprendizaje lineal y aplicativo y brega por la idea de un aprendizaje que se construye desde el hacer mismo y en diálogo con diferentes campos del saber. Posicionar la intervención desde esta premisa implica corrernos de lo tradicional y abrirnos al desafío de otro modos de enseñar y aprender. Las PEEE se integran al programa de las asignaturas que conforman las carreras de grado, al decir de Inés Dussel:

"Estas actividades no son extracurriculares, filantrópicas, marginales, sino que se anclan en ejes estructurales que hacen a la organización pedagógica (...).En su relación con el curriculum, con la enseñanza y el aprendizaje, aparece una idea fuerte de saberes socialmente productivos" (2007:281).

Estas cuestiones que se plantean no pueden perder de vista el contexto al que pertenecemos como universidad, un contexto lleno de desigualdades, donde intereses económicos priman por sobre los valores humanos, por lo que esto nos permitiría decir que la construcción del conocimiento no se puede hacer al margen de ese contexto sociocultural.

\section{Las estrategias institucionales de la UNL de integración de la docencia y la extensión}

Los retos que se presentan tienen que ver con promover y fortalecer espacios y procesos de articulación y generación de acciones colaborativas en estrecha interacción con todas las unidades académicas, hacia adentro de la Universidad y la sociedad en su conjunto, hacia afuera de ésta. 


\author{
G6 \\ un aprendizaje se construye \\ desde el hacer mismo y en diálogo \\ con diferentes campos del saber
}

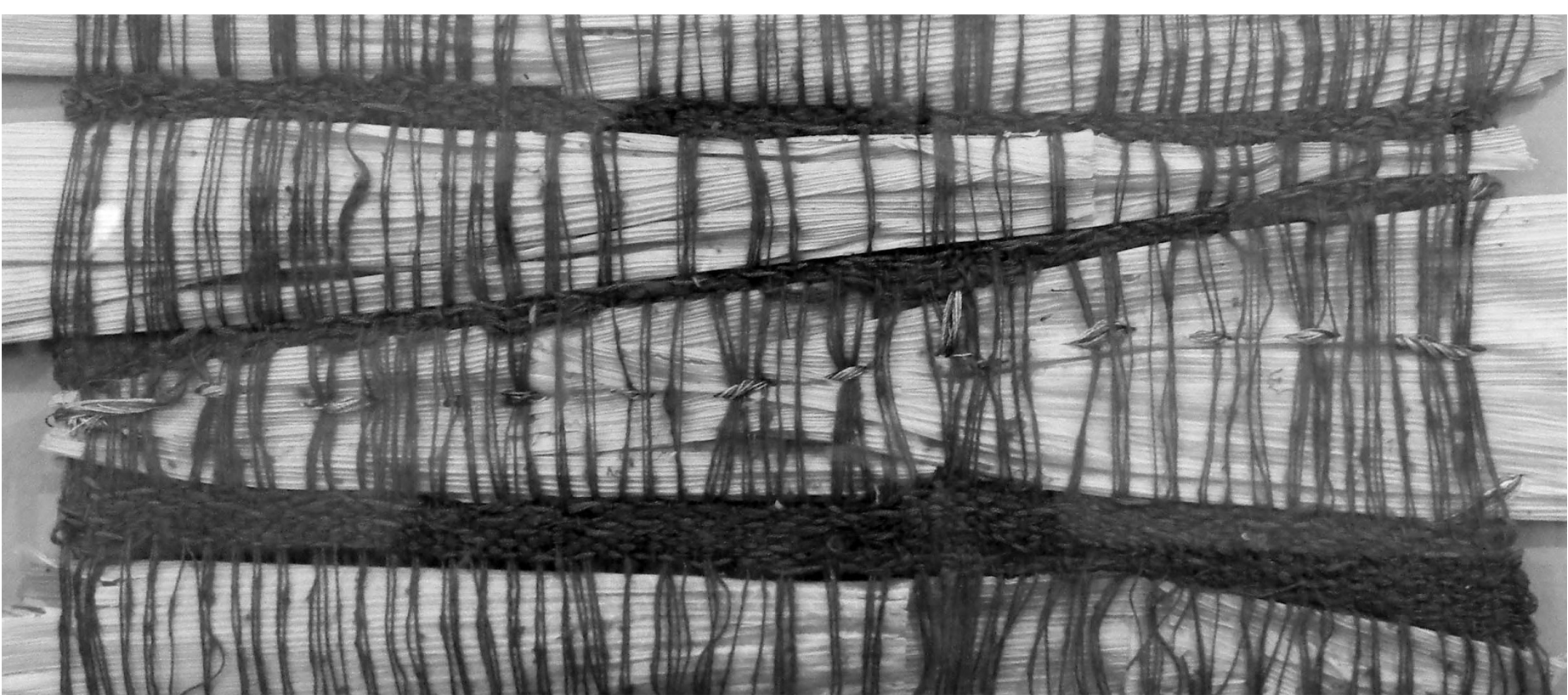

(c) Cecilia lucc

En este sentido es que el trabajo del área de Incorporación Curricular de la Extensión, remite al Plan de Desarrollo Institucional-UNL (2010-2019) y toma como referencia lo que éste expresa cuando dice: "la integración entre la Extensión y la Enseñanza nos posiciona desde una perspectiva en la que los saberes culturales y los conocimientos académicos-científicos se ponen en juego con otros saberes y conocimientos que posee la sociedad, lo que posibilita analizar y abordar los problemas a partir de una concepción y comprensión de la complejidad." (20)

En virtud de esta afirmación, uno de los objetivos del PDI plantea "promover una mayor institucionalización de la extensión, integrada con la docencia, involucrando a toda la comunidad universitaria" y da lugar al Plan y Acción "Fortalecimiento de la Educación Experiencial en el currículo universitario", que busca promover la institucionalización de las prácticas de extensión, enmarcadas pedagógicamente en el enfoque de la educación experiencial. Según Alicia Camilloni, la educación experiencial es considerada una estrategia de enseñanza con enfoque holístico, que está destinada a relacionar el aprendizaje académico con la vida real. Con ese fin, propone al alumno realizar actividades en las que, a partir de esa conexión con la práctica, desarrolle experiencias que resultan de poner a prueba en situación auténtica las habilidades y los conocimientos teóricos que posee, evalúe sus consecuencias, enriquezca esos conocimientos y habilidades, identifique nuevos problemas y fije prioridades en cuanto a las urgencias de su solución.

A partir de esto, Brown (1993) afirma:

"El compromiso social inherente al profesional universitario no puede pensarse como un acto Ad Hoc a la formación disciplinar sino que debe ser el resultado de la apropiación que se materializa a través de los procesos intersubjetivos de comprensión situados. Estos procesos son factibles por y en las prácticas colectivas, en la escucha, el diálogo y la negociación permanente de significados entre los actores involucrados. (...) participar en actividades de extensión es el impulso inicial para movilizar procesos de formación auténtica." (Citado por Rafaghelli, 2013:23) 
Teniendo en cuenta esta conceptualización de las prácticas de extensión, los estudiantes deben disponer de una gran variedad de oportunidades y materiales para promover el aprendizaje "profundo", caracterización empleada por la profesora Alicia Camilloni en su conferencia "Las prácticas académicas en espacios socioculturales, productivos y/o comunitarios. Pensando la educación experiencial", y ofrecer suficiente evidencia en la que basar el conocimiento. Por lo que, a partir de la reconstrucción de las vivencias, reflexiona acerca de su propio desempeño.

Como dice Litwin:

"La significatividad social se construye al entender los conocimientos en una trama de relaciones o vínculos, tal como se presenta en el mundo científico y en el acontecer cotidiano (...); Poner en juego los saberes y los conocimientos que posee la sociedad implica integrar. (...) "...en las manifestaciones de los docentes, significa que los estudiantes doten de sentido al conocimiento adquirido." $(2008: 73,75,76)$

A modo de síntesis, se puede decir que los diseños de los currículos generalmente se proponen relacionar el conocimiento, sus orígenes, los valores y cómo se vinculan con otros temas, pero también que en esa relación es posible que existan dos propuestas diferentes. Como dice Litwin (2008):

"integrar los contenidos diversos en las propuestas de enseñanza o pedir a los estudiantes que, una vez que comprendieron, integren lo nuevo aprendido con conocimiento de la realidad social o personal que ya poseían. En la primera, se trata de organizar los contenidos para proveer la integración. En la segunda, se trata de una exigencia referida a los procesos cognitivos de los estudiantes." (73)

\section{Síntesis de nuestra experiencia}

En virtud de esto que se viene diciendo, queremos compartir nuestra experiencia institucional: el Plan y Acción (en adelante PyA) "Fortalecimiento de la Educación Experiencial en el currículo universitario", que plantea el reto de diseñar estrategias, dispositivos y acciones permitan, a través del consenso con las unidades académicas, docentes, secretarios y estudiantes, la construcción y apropiación por parte de la comunidad educativa de las prácticas de extensión desde este enfoque. El propósito general es consolidar la capacidad crítica y el compromiso social de los estudiantes, futuros graduados y docentes de la UNL. Con relación a ello, se vienen llevando a cabo varias acciones para alcanzar las metas propuestas.

De la acción "Diseñar y ejecutar en forma conjunta las Secretarías Académicas y de Extensión de la Universidad, consejos asesores académicos y de extensión y las unidades académicas, dispositivos institucionales que posibiliten el desarrollo de las primeras prácticas de extensión de educación experiencial" se lograron las siguientes metas: se elaboró, en conjunto con la Secretaría Académica y las unidades académicas, el documento "Experiencia Institucional. Documento General de Prácticas de Extensión de Educación Experiencial" (en adelante PEEE), a los fines de dar un marco a estas prácticas en la UNL, avalado por el Consejo Superior en julio de 2014. Se identificaron los equipos docentes interesados en presentar propuestas de PEEE. Se acordó con las unidades académicas (UA) realizar en el marco del presente PyA convocatorias institucionales que desde la Secretaría de Extensión, a través del área de Comunicación, se difundieron para que cada UA identifique a los equipos docentes que luego llevaron adelante las experiencias de PEEE. Actualmente, nos encontramos en la quinta convocatoria, con un total aproximado de 90 prácticas. También podemos decir que, sobre un total de $10 \mathrm{UA}$, el $60 \%$ de las carreras ha presentado PEEE.

Otra acción es "Identificar recursos humanos internos y externos que posibiliten la organización y efectivización de la capacitación a docentes y estudiantes de la UNL respecto de estas prácticas". Pensada ésta como potenciadora de espacios de reflexión y acompañamiento a los docentes y estudiantes, hasta el momento se realizaron diferentes talleres con una participación que denota el compromiso asumido por los equipos de trabajo tanto pertenecientes a las propuestas presentadas como a las prácticas realizadas. Creemos que el acompañamiento que la institución hace a sus docentes y estudiantes, a partir de la capacitación y la posibilidad que ellos tienen de cursar la asignatura electiva "Extensión Universitaria: prácticas en el territorio" permiten el alcance de las metas propuestas: docentes capacitados en el enfoque de educación experiencial y estudiantes formados en prácticas de educación experiencial.

Finalmente, con relación a "Acordar criterios de evaluación entre las Secretarías de Extensión y Académica de la UNL junto a las 
diferentes unidades académicas a los fines de validar el proceso de implementación y acreditación de las mismas", podemos decir que nos encontramos en el proceso de recopilación de toda la información registrada en el transcurso del presente PyA con el fin de poder lograr la meta asumida. En este sentido, fuimos diseñando instrumentos de sistematización y promoviendo espacios de socialización de las prácticas llevadas a cabo.

Para esta manera de entender la educación, el conocimiento transcurre dentro de los sistemas de actividad que se desenvuelven social, cultural e históricamente, e involucran a personas que se vinculan de manera múltiple y heterogénea.

Cabe destacar que en todo este proceso, en el que los estudiantes transitarán permanentemente por fases de discusión, investigación, acción y fundamentalmente reflexión acerca de lo actuado, son muy importantes el acompañamiento, la orientación, la guía, la ayuda y la supervisión de estas actividades. Por ello se debe contar con la presencia de un equipo docente que sea quien oriente el aprendizaje a partir de la extensión.

Sin dejar de considerar o de reconocer que cada docente optará por lo más adecuado a la hora de pensar en el desarrollo del proceso que se plantea, y teniendo en cuenta, como dice Litwin, que raramente las estrategias están disociadas o aisladas del contenido. Este desafío tiene que ver con explorar de manera diferente nuestras prácticas de enseñanza, y en este caso podríamos decir desde el centro del curriculum, de modo de poder alentar la indagación, la reflexión, la observación sistemática y el espíritu científico.

"La enseñanza requiere que provoquemos a nuestros estudiantes para que realicen diferentes actividades con el objeto de aprender, dada nuestra certeza de que los alumnos aprenden más y mejor cuando participan activamente en la organización y búsqueda de relaciones entre la información nueva y la ya conocida, y no solo cuando reciben nueva información." (Litwin, 2008)

\section{Referencias bibliográficas}

Camilloni, A. (2010). Material Curso de Posgrado. "Las prácticas académicas en espacios socio culturales, productivos y/o comunitarios. Pensando la educación experiencial". Co-organizado por Facultad de Humanidades y Ciencias, Secretarías Académica y de Extensión UNL (Área de Incorporación Curricular de la Extensión). Camilloni A. (2013). La inclusión de la educación experiencial en el curriculum universitario. En Menéndez G. y otros, Integración docencia y extensión. Otra forma de enseñar y aprender. Santa Fe: Ediciones UNL.

Carballeda, A. (2002). La intervención en lo social. Buenos Aires: Paidós.

De Alba, A. (1995). Curriculum, mitos y perspectivas. Buenos Aires: Miño y Dávila Editores.

Dewey, J. (1938). Cómo pensamos. Nueva exposición de la relación ente el pensamiento reflexivo y proceso educativo. Barcelona: Paidós.

Dussel, I. (2007). Comentarios de las experiencias ganadoras del premio presidencial. "Escuelas solidarias" 2005. Antología 1997-2007. Seminarios internacionales. Aprendizaje y Servicio Solidario. Buenos Aires: Eudeba.

Freire, P. (1973). ¿Extensión o Comunicación? La concientización en el medio rural. Montevideo: Siglo XXI y Tierra Nueva.

Kessler y otros (2016). La intervención social en el marco de la Extensión Universitaria. UNL. Mimeo.

Kolb, D. (1984). Experiential Learning. Expeience as the source of learning an development. New Jersey: Prentice-Hall, Inc. A Simon \& Schuster Company. Englewood Cliffs.

Litwin, E. (2008). El oficio de enseñar. Condiciones y contextos. Buenos Aires: Paidós. Matus, S. T. (2005). Apuntes sobre intervención social. Recuperado de: https://trabajosocialucen.files.wordpress.com/2012/04/apuntessobreintervencionsocial.pdf

Rafaghelli, M. (2013). La dimensión pedagógica de la extensión. En Menéndez G. y otros, Integración docencia y extensión. Otra forma de aprender y de enseñar. Santa Fe: Ediciones UNL.

Sousa Santos, B. de (2008). La Universidad en el siglo XXI. Para una reforma democrática y emancipadora de la universidad. Buenos Aires: Miño y Dávila editores. Temporetti, F. (2006). Prácticas Educativas: entre lo individual y lo sociocultural. Breve ensayo sobre los conocimientos psicológicos en la enseñanza. Itinerarios Educativos, la Revista del INDI, 1, 89-102. Santa Fe: Ediciones UNL. UNL (2010). Plan de Desarrollo Institucional (2010-2019). 